Department of Materials, University of Oxford

\title{
Comparison of Experiments and Theories for Plastic Deformation in thermally processed GaAs Wafers
}

Different types of dislocation bundles were identified in thermally processed (001) GaAs wafers. Synchrotron based single crystal X-ray transmission topography, scanning infrared polariscopy, visible light interferometry, standard Nomarski microscopy, and Makyoh topography had been applied for this purpose and allowed for a classification of the dislocation bundles into one distinct majority and a generic minority type. The currently accepted theories are briefly discussed and it is shown that their disagreement with the core of the experimental observations is due to oversimplifications. A new theory is finally presented in a concise manner and its excellent agreement with all of the available experimental evidence is demonstrated.

\section{Introduction}

It is well known that heat treatment induced plastic deformation of GaAs wafers is a key factor that reduces the yield of electronic devices in manufacturing processes on an industrial scale [KIYAMA et al., TATSUMI et al., SAWADA et al., YAMADA et al.]. Currently, advanced industrial processes are being geared towards processing of six-inch diameter GaAs wafers.

Our recent X-ray topographic survey showed that a quite common, radiatively heated, non In-bonded sample holder design caused severe plastic deformation in two-inch diameter GaAs (001) substrates when they were heated in a molecular beam epitaxy (MBE) growth chamber up to about $650{ }^{\circ} \mathrm{C}$ (i.e. up to the commonly applied surface oxide desorption temperature which usually precedes any epitaxial growth) and cooled down to room temperature [MÖCK et al., MÖCK]. Unintentional plastic deformation occurred in all three investigated MBE machines, which were of different make, but we overcame the technical problem by means of modifications to the sample holder of a user built MBE machine [MÖCK and SMITH]. 
At present, however, there is no theory available that can describe the experimental observations including the spatial distribution of the majority of the dislocation bundles satisfactorily. It is only the so called minority type dislocation bundles and their predominance at the peripheral areas of the orientation flats that are reasonably well accounted for by the currently accepted models [KIYAMA et al., SAWADA et al., YAMADA et al.].

As stated by INDENBOM, one has to expect from the experimentally observed density of thermally induced dislocations (i.e. $\approx 510^{3} \mathrm{~cm}^{-2}$, averaged over the whole wafer) in two-inch diameter GaAs wafers [MÖCK et al.] that the averaged absolute values of the resolved thermal shear stresses will be of the order of magnitude of a certain critical thermal stress for dislocation generation. The consequence of this is that the spatial correlation between the distribution of dislocations and the distribution of the resolved thermal shear stresses will be blurred by inhomogeneities such as surface irregularities, freshly introduced dislocations, and pre-existing defects. While the most comprehensive of the currently accepted models [SAWADA et al.] relies entirely on such a correlation, our new model includes influences of such inhomogeneities.

The first aim of this paper is to compare theoretical predictions of the old theories [KIYAMA et al., SAWADA et al., YAMADA et al.] and of our new model with a variety of experimental observations, demonstrating an excellent agreement in the latter case. The paper's second aim is to present in a concise manner the concept of our new model, which is applicable to both majority and minority type dislocation bundles in a circular wafer.

\section{Experimental observations}

As Figs. 1a,b show, the plastic deformation up to about $98 \%$ is realised by bundles of dislocations which start at the sample edges around the four <100> peripheral areas. These dislocation bundles glide into the bulk of the wafer following $\langle 110\rangle$ and $\langle-110\rangle$ line directions, and form a pseudo-symmetric, four-fold set in undoped GaAs, as shown in Figs. 1a,b, and a two-fold set in Si doped GaAs as shown by MÖCK. This sort of dislocation bundle is named majority type dislocation bundle.

A Burgers vector analysis of the complete pseudo-symmetric set of majority type dislocation bundles showed that there are dislocations with two different Burgers vectors in each of these bundles and that the straight extended segments perpendicular to the wafer normal of all of these dislocations are of the $60^{\circ}$ type 
[MÖCK]. We suggested that dislocations with different Burgers vector in a dislocation bundle pair up, thus, cancelling in effect surface steps and slip lines to some extent and providing effective relief of thermally induced shear strains. Both effects of the proposed pairing up of dislocations have been corroborated by indirect means [MÖCK et al.], comparing images obtained by means of quantitative scanning infrared polariscopy (Figs. 1a, 3a, and 3b), synchrotron based single-crystal X-ray transmission topography (Fig. 1b), Makyoh topography (Fig. 2a), visible-light interferometry (Fig. 2b), and standard Nomarski microscopy.

In addition to majority type dislocation bundles, there can be dislocation bundles of (at least) two minority types with a spatial distribution that is centred at and around <110> peripheral regions [MÖCK]. As Figs. 2a,b show, only minority type dislocation bundles (i.e. only a few percent of all dislocations) can be detected by commonly applied surface inspection techniques since only they form slip line bunches of sufficient height at the free wafer surface. While dislocation bundles of the first minority type tend to be located at or rather close $\left(< \pm 10^{\circ}\right)$ to a $<110>$ pole, dislocation bundles of the other minority types deviate from about $\pm 10^{\circ}$ up to about $\pm 35^{\circ}$ from a $\langle 110>$ pole. Thus, they partially overlap the areas that are occupied by the pseudosymmetric set of majority type dislocation bundles, which typically extends up to about $\pm 25^{\circ}$ around $<100>$ poles [MÖCK et al.]. The experimental evidence from one of KIYAMA's et al., SAWADA's et al. figures shows that there are more and longer slip lines (i.e. minority type dislocation bundles) closer to $\langle 110\rangle$ than to $<100>$.

In agreement with CURIE's symmetry principle, we derived experimentally the point symmetry group 4 $2 \mathrm{~m}$ for the crystallographic parameters of the majority type dislocation bundles in undoped GaAs [MÖCK]. There are representations of a four-fold inversion axis in [001] orientation, two two-fold axes in <100> orientation, and two mirror planes in $\langle 110\rangle$ orientation visible in Figs. 1a,b. Thus, there is, for majority type dislocation bundles, as already stated above, a four-fold pseudo-symmetry, which is centred around <100> peripheral areas.

For a sufficiently high density of minority type dislocation bundles in undoped GaAs (i.e. slip lines at the free wafer surfaces, which have been analysed by means of standard Nomarski microscopy [KIYAMA et al., SAWADA et al.]), a four-fold pseudo-symmetry which is centred around $\langle 110\rangle$ peripheral areas can be observed. This pseudo-symmetry contains representations of exactly the same symmetry elements as stated above for majority type dislocation bundles. For an explanation of the difference of true crystallographic symmetry and pseudo-symmetry, see appendix 1 . 


\section{Theories}

\subsection{Currently accepted models}

\subsubsection{Concept and main prediction}

Both of the currently accepted models [KIYAMA et al., SAWADA et al., YAMADA et al.] use a similar approach to predict whether or not plastic deformation should occur. While SAWADA et al, considered only the eight slip systems with Burgers vectors inclined to $45^{\circ}$ to the surface normal, YAMADA et al. considered all twelve possible slip systems.

The model by SAWADA et al. goes further than the basic ideas by KIYAMA et al. from which it evolved and considers, following JORDAN et al., the differences between eight resolved thermal shear stresses and the critical resolved shear stress to be proportional to the amount of plastic deformation, i.e., in other words, to the dislocation number, density, and length as well as to the height and length of slip lines at the free wafer surface. The sum of all of these eight differences as a function of location over the wafer periphery is supposed to give a prediction of the spatial distribution of the dislocations and their associated slip lines. The maxima of plastic deformation are predicted to occur midway between $\langle 110\rangle$ and $\langle 100\rangle$ peripheral areas [SAWADA et al.].

The model by YAMADA et al. derives the ratio of the maximal resolved thermal shear stresses for glide of dislocations with Burgers vectors that are either inclined at $45^{\circ}$ or perpendicular to the wafer surface normal. This ratio is about 1.2 and leads to the prediction that dislocation glide should commence in peripheral areas midway between $\langle 100\rangle$ and $\langle 110\rangle$. Since the resolved thermal shear stresses are highest at these positions and dislocation glide is supposed to have started just there, the dislocations are predicted to be longest and most numerous midway between $\langle 100\rangle$ and $<110>$ [YAMADA et al.].

\subsubsection{Comparisons of main prediction with experimental results}

Figs. 1a,b and 3b show plain disagreement with the currently accepted models [KIYAMA et al., SAWADA

et al., YAMADA et al.], for the spatial distribution of the majority type dislocation bundles is obviously 
centred around the four $\langle 100\rangle$ peripheral areas. Figs. 2a,b, on the other hand, show only minority type dislocation bundles which are located at positions not far away from the main prediction of these models.

For cases of a low density of slip lines (i.e. minority type dislocation bundles), as presented in Figs. 2a,b, most of the figures given by KIYAMA et al., SAWADA et al., and all of the figures given by YAMADA et al., it is impossible to elucidate the pseudo-symmetry and crystallographic directions with the highest number, density and length of dislocations. For a sufficiently high slip-line density, however, there is a fourfold pseudo-symmetry which is centred around $\langle 110\rangle$ peripheral areas and there ae more minority type dislocation bundles closer to $\langle 110\rangle$ than to $<100\rangle$ [KIYAMA et al., SAWADA et al.]. Obviously, these two pieces of experimental evidence contradict the main theoretical prediction of the currently accepted models as well.

From the viewpoint of basic physical crystallography, a distribution that has its maximum midway between $\langle 110\rangle$ and $\langle 100\rangle$ possesses an eight-fold symmetry. Since, following CURIE's symmetry principle, the intersection of the point symmetry group of the applicable temperature field $(\infty \mathrm{mm})$ with the point symmetry group of the GaAs crystal $(43 \mathrm{~m})$ does not contain an eight-fold symmetry axis, the models by KIYAMA et al., SAWADA et al., YAMADA et al. can only be oversimplifications. This demonstrates clearly that for modelling purposes it is not sufficient to consider only the resolved thermal shear stresses. 


\subsection{New model}

\subsubsection{Concept of new model and simulation conditions}

The new model was developed on the basis of the experimentally derived crystallographic symmetry properties of the pseudo-symmetric set of majority type dislocation bundles (which allowed us to deduce the symmetry properties of the applicable strain and stress tensors [REID]), the calculated resolved thermal shear stresses on the eight actually operating dislocation glide systems, the naturally occurring distribution of dislocation sources, and dislocations pairs as basic constituents of majority type dislocation bundles. For most considerations, the stress distribution during plastic flow is assumed to be independent of the number, density and spatial distribution of freshly introduced dislocations. When needed for the explanation of one particular experimental observation, we will, however, make use of this dependence. The dependence of the stress distribution during plastic flow on grown-in dislocations will, on the other hand, always be neglected. Hooke's law in its isotropic approximation is, as demonstrated by similar calculations for bulk GaAs crystals [JORDAN et al.], considered to be sufficiently accurate for modelling purposes.

In order to demonstrate the predictive power of our model, we are going to perform a simulation for conditions that are quite close to our experiments. In order to do so, we employ Hooke's law to obtain the actually encountered axial stress $\sigma_{\text {axi }}$ from the actually encountered strain $\varepsilon_{\text {axi }}$

$\sigma_{\mathrm{axi}}=\varepsilon_{\mathrm{axi}}\left(\mathrm{c}_{11}+2 \mathrm{c}_{12}\right)$

where the $c_{\mathrm{ij}}$ are the stiffness tensor components. We consider $\varepsilon_{\text {axi }}=\varepsilon_{33}{ }^{\text {act }}$ to be equal to $2 \varepsilon_{33}{ }^{\text {res }}+\varepsilon^{\text {cri }}$, where $\varepsilon_{33}{ }^{\text {res }}=\varepsilon_{\mathrm{xx}}{ }^{\text {res }}=\varepsilon_{\mathrm{yy}}{ }^{\text {res }}$ (which is a consequence of the applicable deformation geometry [REID]) has to be extracted from residual strain maps $\left|\varepsilon_{\mathrm{xx}}-\varepsilon_{\mathrm{yy}}\right|$ such as the one shown in Fig. 3a, see also appendix 2. The

superscripts ${ }^{a c t}$, res , and ${ }^{c r i}$ stand for actually encountered during a thermal treatment, residual at room temperature, and critical for yielding. The subscripts ${ }_{a x i}$, rad, and ${ }_{\text {tan }}$ stand for axial, radial and tangential. As it is common practice, stresses are represented in general by the symbol $\sigma$, resolved shear stresses by the symbol $\tau$, and strains by the symbol $\varepsilon$. 
The elastic strain at the yield point $\left(\varepsilon^{\text {cri }}\right)$ is calculated from the "critical stress for slip defect generation", as given by KIYAMA et al., for (001) oriented GaAs wafers (circular with two orientation flats) in the applicable deformation geometry [REID], see also appendix 2 . We assume further $\varepsilon_{\text {rad }}$ and $\sigma_{\text {rad }}$ to be $5 \%$ of $-\varepsilon_{\tan }$ and $-\sigma_{\tan }$, respectively. In other words, we consider tangential strains and stresses as dominant in all regions close to the margin of the wafer and residual strain maps $\left|\varepsilon_{\text {rad }}-\varepsilon_{\text {tan }}\right|$ such as the one shown in Fig. 3b as an approximation of the spatial distribution of the tangential strain component. Thus with $\varepsilon_{\tan }=\varepsilon_{\tan }{ }^{\text {act }}=2$ $\varepsilon_{\tan }^{\text {res }}+\varepsilon^{\text {cri }}$ (see also appendix 2) and Hooke's law

$\sigma_{\tan }=\left\{\varepsilon_{\tan }+{ }^{v} / \varepsilon_{\mathrm{Exi}}\left(\mathrm{c}_{11}+2 \mathrm{c}_{12}\right)\right\} /\left({ }_{\mathrm{E}}+{ }^{v} /{ }_{20 \mathrm{E}}\right)$

where E: Young's modulus, $c_{\mathrm{ij}}$ : stiffness tensor components, and v: Poisson's ratio we are able to calculate the actually encountered tangential stress for conditions that are quite close to our experimental conditions.

With $\sigma_{\tan }, \sigma_{\text {axi }}$ and $\sigma_{\text {rad }}$ known, the resolved thermal shear stresses $\tau_{\mathrm{i}}$ on the twelve glide systems in a cylindrical co-ordinate system are obtained by:

$\tau_{1,2}=1 / \sqrt{ } 6\left[\sigma_{\text {axi }}-\sigma_{\tan }-\sqrt{ } 2\left(\sigma_{\text {rad }}-\sigma_{\tan }\right) \sin \theta \sin \left(\theta+\pi /{ }_{4}\right)\right] \mid$

for $[0-11](111)$ and $[011](-1-11) ;(3 a)$

$\tau_{3,4}=1 / \sqrt{ } 6\left[\sigma_{\text {axi }}-\sigma_{\tan }-\sqrt{ } 2\left(\sigma_{\text {rad }}-\sigma_{\tan }\right) \sin \theta \sin (\theta-\pi / 4)\right] \mid$

for $[0-11](-111)$ and $[011](1-11) ;(3 b)$

$\tau_{5,6}=\vdash^{1} / \sqrt{ } 6\left[\sigma_{\text {axi }}-\sigma_{\tan }-\sqrt{ } 2\left(\sigma_{\text {rad }}-\sigma_{\tan }\right) \cos \theta \sin (\theta+\pi / 4)\right] \mid$

for $[10-1](111)$ and $[-10-1](-1-11) ;(3 c)$

$\tau_{7,8}=\vdash^{1 / \sqrt{ } 6}\left[\sigma_{\text {axi }}-\sigma_{\tan }+\sqrt{ } 2\left(\sigma_{\text {rad }}-\sigma_{\tan }\right) \cos \theta \sin (\theta-\pi / 4)\right] \mid$

for $[-10-1](-111)$ and $[10-1](1-11) ;(3 d)$

$\tau_{9,10,11,12}=\left.\right|^{1} / \sqrt{ } 6\left(\sigma_{\text {axi }}-\sigma_{\tan }\right) \cos 2 \theta \mid$

for $[-110](111),[110](-111),[1-10](-1-11)$, and [-1-10](1-11); (3e) 
where $\theta$ is an angular parameter that counts from [100], i.e. the formulae for the five independent resolved thermal shear stresses [JORDAN et al.]. Following appendix 3, the resolved thermal shear stresses for dislocation pairs are obtained by adding up the relevant $\tau_{\mathrm{i}}(\theta)$ to form the sums $\Sigma \tau_{\mathrm{ij}}(\theta)$.

The first condition for the existence of a dislocation bundle at an appropriate spatial location is that the resolved thermal shear stress on the applicable glide system is greater than the "critical stress for slip defect generation" [KIYAMA et al.], below referred to as critical thermal stress for dislocation generation. A justification of our usage of this threshold value rather than that of the critical resolved shear stress is given in appendix 4.

As the discussion of the currently accepted theories [KIYAMA et al., SAWADA et al., YAMADA et al.] has shown, effects such as the heterogeneous nucleation of dislocations at surface irregularities can not be neglected. Thus, we consider the spatial distribution of dislocation bundles as obtained from formulae (1), (2) and (3a) - (3d) to be modified by the naturally existing distribution of dislocation sources, which is highest at $<100>$ peripheral areas (appendix 5).

\subsubsection{Predictions for a circular GaAs wafer at $650{ }^{\circ} \mathrm{C}$ and comparison to experimental results}

Since the surface oxide desorption step took place in our experiments at the highest temperature of the whole thermal cycle (and because we do not known when precisely in the thermal cycle the plastic deformation occurred), we perform for simplicity calculations for this particular temperature only. At $650{ }^{\circ} \mathrm{C}$, the relevant elastic parameters for GaAs are $\mathrm{E}=78.351 \mathrm{GPa}, \mathrm{c}_{11}=108.88 \mathrm{GPa}, \mathrm{c}_{12}=48.9 \mathrm{GPa}$, and $\mathrm{v}=$ 0.3105 [INSPEC]. At the same temperature, the experimentally determined critical thermal stress for dislocation generation under the applicable deformation geometry [REID] is 5.46 MPa [KIYAMA et al.].

Assuming a tangentially uniform residual strain distribution, formulae (1), (2) and (3a) - (3d) give for the

outermost margin of the residual strain annulus (i.e. $4.7510^{-5}$, see Fig. $3 b$ ) two curves for the resolved thermal shear stresses $\tau_{i}(\theta), i=1,2,5$, and 6 , over an angular parameter $(\theta)$ measured from the [100] direction (Fig. 4). Calculations for the other four operating slip systems (i.e. $\mathrm{i}=3$, 4, 7, and 8; graph not shown) lead to an analogous graph with an angular shift of $90^{\circ}$. 
In Fig. 4, there are only four small angular regions where $\tau_{1}(\theta), \tau_{2}(\theta), \tau_{5}(\theta)$, and $\tau_{6}(\theta)$, possess values that are bigger than the experimentally observed threshold value for dislocation generation. This explains that minority type dislocation bundles can glide in certain peripheral areas after they have nucleated by some mechanism.

Fig. 4 shows in agreement with Figs. 1a,b and 2a,b that the area where minority type dislocations can exist ranges from about $10^{\circ}$ to about $35^{\circ}$ around $\langle 110\rangle$ peripheral areas. The fact that our model does not allow for dislocation bundles closer than about $10^{\circ}$ to a $\langle 110\rangle$ pole indicates that dislocation bundles of the so called first minority type [MÖCK] are not accounted for by our model which assumes a perfectly circular wafer. Thus, we believe that dislocations bundles of the first minority type are due to the influence of the crystal orientation flats on the distribution of thermal stresses.

The maxima in the $\tau_{i}(\theta)$ curves occur at positions of $\pm \pi / 8$ around each $\langle 100\rangle$ direction, i.e. midway between $\langle 100\rangle$ and $\langle 110\rangle$, as obtained previously by the rival models [KIYAMA et al., SAWADA et al., YAMADA et al.]. We suggest, however, that the dependence of the stress distribution during plastic flow on the spatial distribution and number density of freshly introduced majority type dislocation bundles causes more minority type dislocation bundles closer to $\langle 110\rangle$ than to $\langle 100\rangle$, as shown in one of the figures by KIYAMA et al., SAWADA et al..

This dependence is neglected by KIYAMA et al., SAWADA et al., YAMADA et al. and not accounted for by calculations based solely on formulae (3a) - (3d). The above assumption is, however, quite reasonable since there are many more majority type dislocation bundles than minority type dislocation bundles, as shown in Figs. 1a,b and 2a,b, and there are peripheral areas of high strain close to $\langle 110\rangle$ directions that can not, partly for geometrical reasons, be relaxed by majority type dislocation bundles. If majority type dislocation bundles were to appear first during plastic flow, their impact on the relaxation of regions around $\langle 110\rangle$ would be to modify the distribution of the strains in a manner that favours the existence of minority type dislocation bundles closer to $\langle 110\rangle$ than to $\langle 100\rangle$.

The startling conclusion from Fig. 4 is, however, if dislocation pairs would not exist a few minority type dislocation bundles would be all that is possible to relax the thermally induced strain. If we, however, take dislocation pairs into account, i.e. if we, in other words, allow for dislocation interactions contrary to the rival models by KIYAMA et al., SAWADA et al., YAMADA et al., the situation changes completely. 
As Fig. 4 shows, the resolved thermal shear stresses $\tau_{1}(\theta)+\tau_{5}(\theta)=\tau_{2}(\theta)+\tau_{6}(\theta)$ for dislocation pairs are above the threshold for dislocation generation for nearly all angular regions. Thus, formation and glide of dislocation pairs is possible nearly everywhere at the wafer edge, but the places where it will actually happen depend on the naturally occurring distribution of surface irregularities around $<100>$ peripheral areas which act as dislocation sources (see Figs. 1a,b). These surface irregularities will modify the critical thermal stress for dislocation generation locally, which is in reality not rigidly fixed anyway, as discussed above. This allows for both constituents of a dislocation pair to be generated in sufficient numbers. The resolved thermal shear stress curves $\Sigma \tau_{15}(\theta)$ and $\Sigma \tau_{26}(\theta)$ in Fig. 4 indicate, as shown in Figs. 1a,b, that the further away a dislocation bundle of the majority type nucleates from an exact $<100>$ peripheral position, the shorter it will be.

The angular range of majority type dislocation bundles is, as Fig. 4 shows in agreement to Figs. 1a,b, about $\pm 22^{\circ}$ around $<100>$ directions. Since $\Sigma \tau_{\mathrm{ij}}\left(I_{2}+\delta\right)=\Sigma \tau_{\mathrm{ij}}\left(/_{2}-\delta\right)$, majority type dislocation bundles can, in agreement to Figs. 1a,b, exist on both sides (i.e. $\pm \delta$ ) around any $\langle 100\rangle$ direction as a consequence of both the applicable deformation geometry [REID] and the existence of dislocation pairs.

A two-fold symmetry of both minority and majority type dislocation bundles is directly depicted in Fig. 4. Taking into account the complementary graph for the other four actually operating slip systems, we obtain for undoped GaAs a four-fold symmetry for both minority and majority type dislocation bundles, see Fig. 1a,b.

Majority type dislocation bundles are not only capable of running in two perpendicular $\langle 110\rangle$ directions in each $\langle 100\rangle$ peripheral area (see Figs. 1a,b), but will, as Fig. 4 shows, also experience the same resolved thermal shear stresses $\Sigma \tau_{\mathrm{ij}}(\theta)=\Sigma \tau_{\mathrm{kl}}\left(\theta+{ }^{\pi} / 2\right)$. Thus, they will be of about the same length (see Figs. 1a,b) as long as the material properties that determine the impediment to dislocation glide are isotropic parallel to the $<110\rangle$ and $\langle-110\rangle$ directions. This is not the case for Si doped GaAs [YONENAGA and SUMINO], and as a consequence $\Sigma \tau_{15}(\theta)=\Sigma \tau_{26}(\theta) \neq \Sigma \tau_{37}\left(\theta+{ }^{\pi} / 2\right)=\Sigma \tau_{48}\left(\theta+{ }^{\pi} / 2\right)$ becomes an expression of a two-fold pseudosymmetry, as shown in one of the figures of the paper by MÖCK. For more information on the difference between true crystallographic symmetry and pseudo-symmetry for plastically deformed, undoped and $\mathrm{Si}$ doped GaAs, see appendix 1.

Fig. 4 demonstrates clearly that there will be many more majority type dislocation bundles than minority type dislocation bundles. The theoretical majority type to minority type dislocation bundle ratio is obtained from the ratio of the areas underneath the $\tau_{\mathrm{i}}(\theta)$ and $\Sigma \tau_{\mathrm{ij}}(\theta)$ curves that are above the critical thermal stress for 
dislocation generation. With this ratio being about 20 and taking into account the naturally occurring distribution of dislocation sources, which favours regions around $\langle 100\rangle$, agreement with the experimental results, given by MÖCK and shown in Figs. 1a,b, is obtained.

For a residual strain of about $2.510^{-5}$, model calculations predict that glide of majority type dislocation bundles from $<100>$ peripheral areas ceases since the resolved thermal shear stresses on dislocation pairs fall below the critical thermal stress for dislocation generation. As Fig. 3b shows, this is in good agreement to the experimental observations.

Contrary to the predictions by YAMADA et al., the four slip systems that involve screw dislocations are, as the experiments [MÖCK] and calculations using formula (3e) show, not activated. This is because their resolved thermal shear stresses $\left(\tau_{9-12}(\theta)\right)$ are for all experimentally observed residuals strain levels (Fig. 3b) well below the critical thermal stress for dislocation generation. The absence of dislocations in the glide systems $\mathrm{i}=9-12$ is no coincidence, but a consequence of the applicable form of the strain and stress tensors, i.e. the deformation geometry in other words [REID].

\section{Conclusions}

The concept of a superior model for plastic deformation of thermally processed circular semiconductor wafers of the sphalerite structure has been presented. The sample holder induced dislocation slip in (001) oriented GaAs substrates during a standard molecular beam epitaxial growth cycle has been used to demonstrate the excellent agreement of the new theory with the whole range of experimental observations. This agreement suggests strongly that the concept of the new semi-quantitative model is essentially correct and that dislocation pairs form the basic constituents of majority type dislocation bundles. The currently accepted models, however, have been proven to represent oversimplifications. 

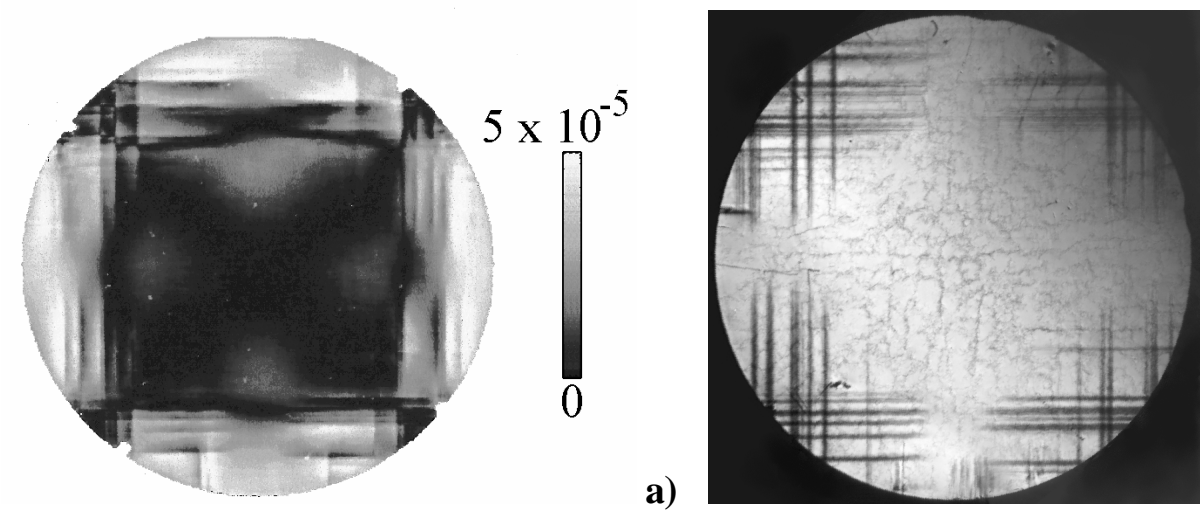

b) $\uparrow[-1-10]$

Fig. 1: "Transmission images" of the sample that is shown as well in Figs. 2a,b and 3a,b; taken from MÖCK et al.; a) left side, Scanning infrared polariscopy map of residual shear strains (i.e. $2\left|\varepsilon_{\mathrm{xy}}\right|, 1.3 \pm 0.1 \mu \mathrm{m}$ wavelength); b) right side, synchrotron based single crystal X-ray transmission topogram (0-22) reflection, $0.13 \mathrm{~nm}$ wavelength). The strain quantifying marker is, as in all other polariscopy maps, linear. As in all other experimental Figs. of this paper, the major flat, i.e. [-1-10] direction, points up towards the top of the page and the whole two-inch diameter wafers are depicted (except for small mounting and imaging artefacts such as superimposed images of dust particles in Fig. 1a). Both majority and minority type dislocation bundles are visible. 


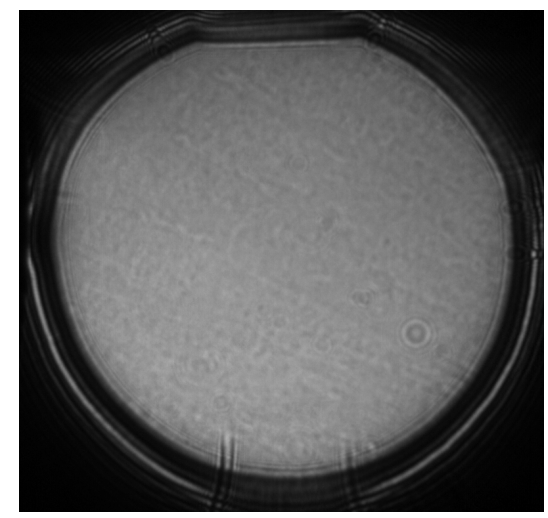

a)

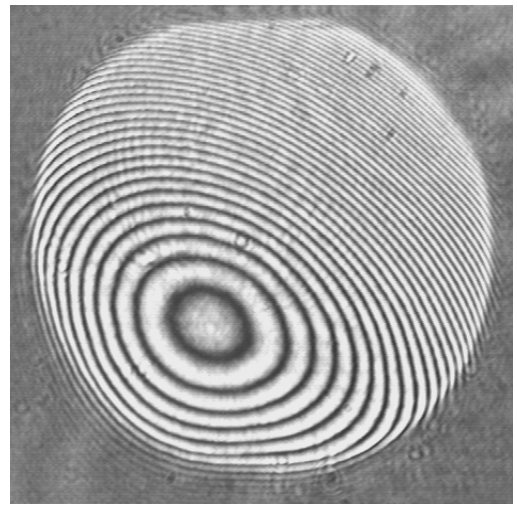

b) $\uparrow[-1-10]$

Fig. 2: "Surface" images of the sample that is shown as well in Figs. 1a,b and 3a,b; 1a taken from MÖCK et al., 2b previously unpublished; a) left side, Makyoh topogram (red He-Ne laser) b) right side, Visible light interferogram (632.8 nm wavelength, courtesy: Dr. Z. Laczik, Department of Engineering Science, University of Oxford). Only minority type dislocation bundles are visible. 

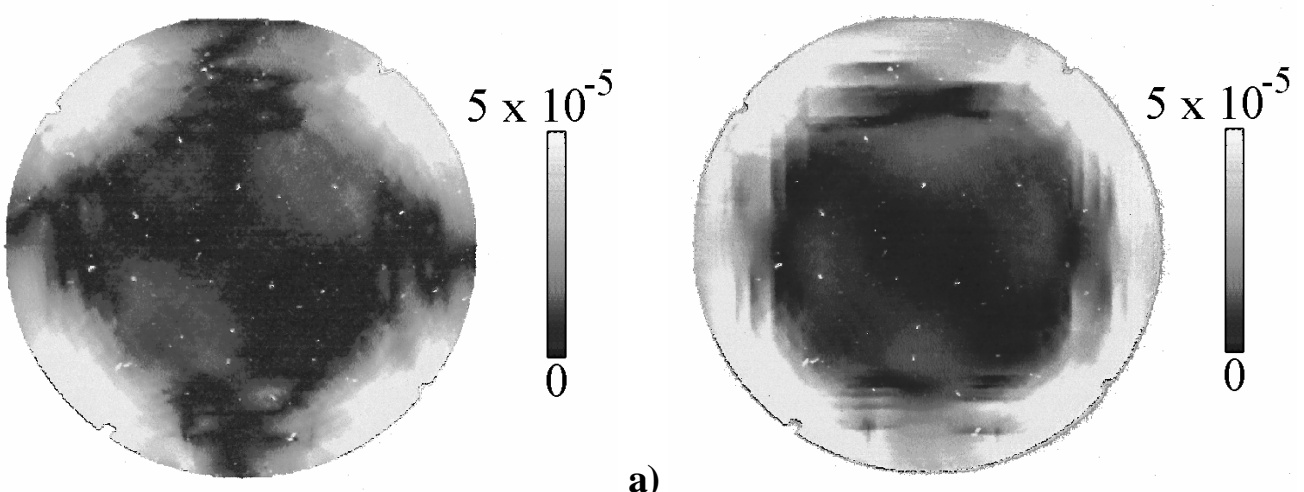

b) $\uparrow[-1-10]$

Fig. 3: Complementary scanning infrared polariscopy maps of the sample that is shown as well in Figs. 1a,b and 2a,b; taken from MÖCK et al.; a) left side, Scanning infrared polariscopy map of the absolute values of the differences in the residual tensile strains parallel to the $\langle 100\rangle$ axes, i.e. $\left|\varepsilon_{\mathrm{xx}}-\varepsilon_{\mathrm{yy}}\right|$; b) right side, Scanning infrared polariscopy map of the absolute values of the differences in residual tensile strains parallel to the radial and tangential directions (in a cylindrical co-ordinate system). Note that Figs. 1a and 3a add up to form Fig. 3b, since $\varepsilon_{\mathrm{rad}}-\varepsilon_{\mathrm{tan}} \mid=\left\{\left(\varepsilon_{\mathrm{xx}}-\varepsilon_{\mathrm{yy}}\right)^{2}+\left(2 \varepsilon_{\mathrm{xy}}\right)^{2}\right\}^{1 / 2}$ [YAMADA]. Thus, both majority and minority type dislocation bundles are visible in Fig. 3b, although not as clearly as in Fig. 1a. 


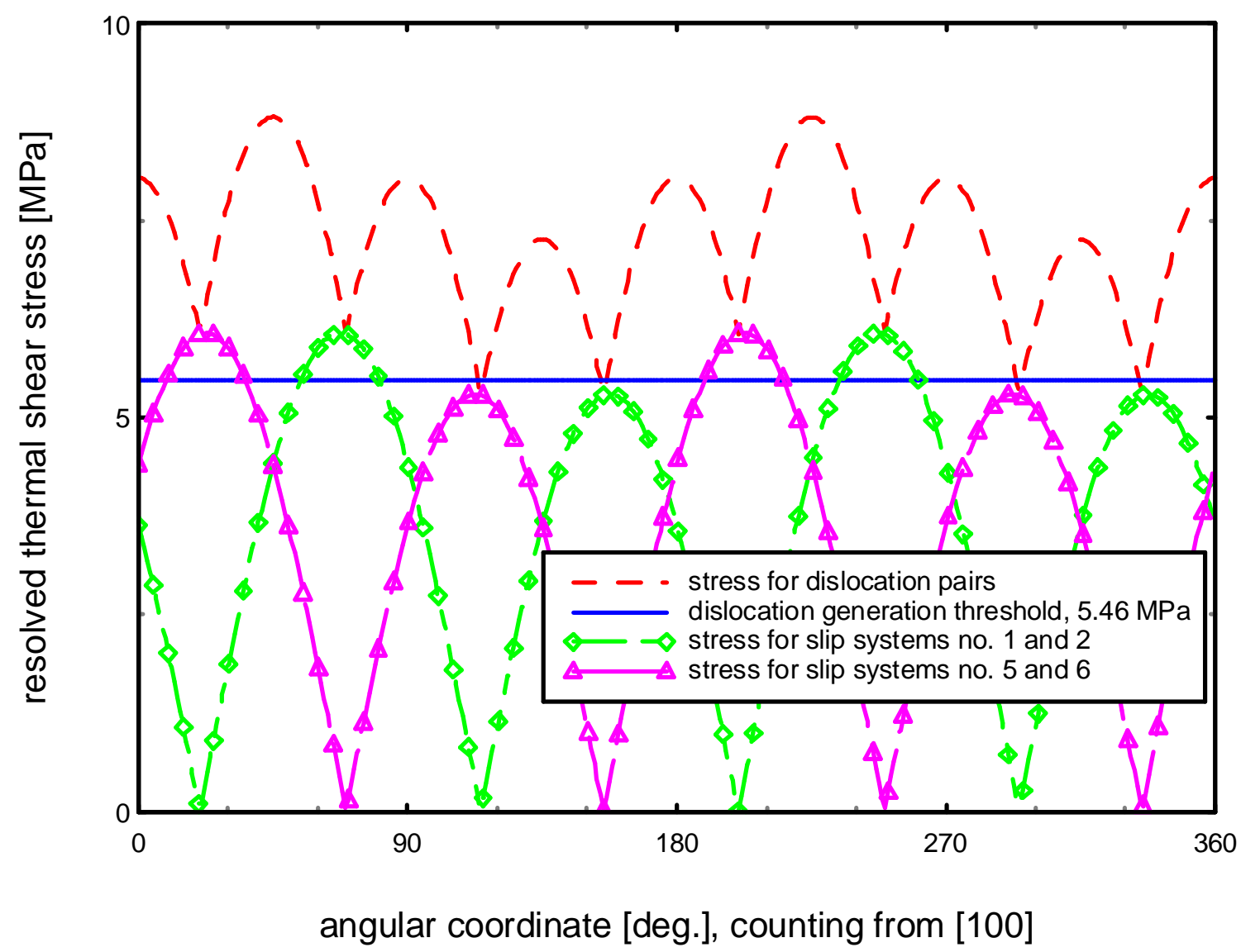

Fig. 4: Predicted resolved thermal shear stresses $\tau_{1}(\theta), \tau_{2}(\theta), \tau_{5}(\theta), \tau_{6}(\theta), \Sigma \tau_{15}(\theta)$, and $\Sigma \tau_{26}(\theta)$ as function of an angular coordinate that counts from [100]. The four slip systems are: no. 1: [0-11] (111), no. 5: [10-1] (111), no. 2: [011] (-1-11), no. 6 [-10-1] (-1-11); the two dislocation pairs are: no. $1+5$ and 2+6. 


\section{Acknowledgements}

We thank Drs. G. R. Booker and R. E. Rudd (Department of Materials, Oxford University) for careful reading of the manuscript. Previous experimental collaboration with Dr. Z. Laczik (Department of Engineering Science, Oxford University), Prof. M. Yamada, Dr. M. Fukuzawa (both Department of Electronics and Information Science, Kyoto Institute of Technology), Prof. B. K. Tanner (Department of Physics, Durham University), Dr. G.W. Smith (Defence Research and Evaluation Agency, Malvern), and Dr. M. Herms (Fraunhofer Institute of Non-destructive Testing, Dresden) on the subject of this paper is gratefully acknowledged. The experimental work at the U.K. Synchrotron Radiation Source, Daresbury Laboratory, was financially supported by the Engineering and Physical Research Council as "Direct Access" projects (No.: 30047 and 31098). 


\section{References}

ALEXANDER H., HAASEN, P.: Solid State Phys. 22 (1968) 27.

CURIE P.: J. Physique 3 (1894) 393.

BILLIG E.: Proc. Royal. Soc. A235 (1956) 37.

FITZGERALD E. A, WATSON G. P., PROANO R. E., AST D. G., KIRCHNER P. D., PETTIT G. D., WOODALL J. M.: J. Appl. Phys. 65 (1989) 2220.

HEY R., WASSERMEIER M., BEHREND J., DÄWERITZ L., PLOOG K., RAIDTH H.: J. Cryst. Growth 154 (1995) 1.

INDENBOM V.L.: Kristall und Technik 14 (1979) 493.

INSPEC, Properties of Gallium Arsenide, The Institution of Electrical Engineers, London, New York, 2nd Edition, 1990, Chapter 1.

JORDAN A. S., CARUSO R., von NEIDA A. R.: Bell System Technical Journal 59 (1980) 593.

KIYAMA K., TAKEBE T., FUJITA K.: Inst. Phys. Conf. Ser. No. 155 (1997) 945.

MÖCK P., FUKUZAWA M., LACZIK Z., SMITH G. W., BOOKER G. R., YAMADA M., HERMS M., TANNER B.K.: Inst. Phys. Conf. Ser. No. 164 (1999) 67.

MÖCK P., SMITH G.W.: submitted to Cryst. Res. Technol.

MÖCK P.: submitted to J. Cryst. Growth

MÖCK P.: submitted to J. Appl. Cryst.

REID C. N.: Deformation Geometry for Materials Scientists, Pergamon Press, Oxford, New York, Toronto, Sydney, Braunschweig 1973.

SAWADA S., YOSHIDA H., KIYAMA M., MUKAI H., NAKAI R., TAKEBE T., TATSUMI M., KAJI M., FUJITA K.: Technical Digest - IEEE GaAs Integrated Circuit Symp. 74 (1996) 50.

TATSUMI M., HOSOKAWA Y., IWASAKI T., TOYODA N., FUJITA K.: Mater. Sci. Engin. B28 (1994) 65.

VÖLKL, J: "Stress in the Cooling Crystal", in "Handbook of Crystal Growth 2, Bulk Crystal Growth, Part B: Growth Mechanisms and Dynamics”, pp. 823 - 874, North-Holland, Amsterdam, London, New York, Tokyo, 1994

YAMADA M., Rev. Sci. Instrum. 64 (1193) 1815.

YAMADA M., FUKUZAWA K., ITO K.: Inst. Phys. Conf. Ser. No. 155 (1997) 901. 
YONENAGA I., SUMINO K.: J. Cryst. Growth 126 (1993) 19. 


\section{Appendices}

\section{Difference between true crystallographic symmetry and pseudo-symmetry}

The difference between true crystallographic symmetry and either four-fold or two-fold pseudo-symmetry is due to both, the spatially not well defined nature of dislocation nucleation and possible multiplication events as well as anisotropic impediment to dislocation glide, as observed in the case of Si doped GaAs [YONENAGA and SUMINO].

For undoped GaAs, the symmetry elements that are showing up as pseudo-symmetry in Figs. 1a,b and $3 b$ and sketches of the slip line distribution (as determined by standard Nomarski microscopy [KIYAMA et al.], [SAWADA et al.]) are, a four-fold inversion axis in [001] orientation, two two-fold axes in <100> orientation, and two mirror planes in $\langle 110\rangle$ orientation. Together with the always present symmetry element 1 , these symmetry elements form the point symmetry group $42 \mathrm{~m}$.

Since dislocation glide properties along <110> and <-110> directions are not equivalent in Si doped GaAs due to anisotropic impediment, the two-fold axes, which are supposed to transform $<110>$ directions into <$110>$ directions, are lost. This reduces the four-fold inversion axis to a two-fold axis and the remaining symmetry elements are the two mirror planes in $\langle 110\rangle$ orientation (as well as the always present symmetry element 1). These symmetry elements do not form a complete point group, revealing that they refer only to a pseudo-symmetry. Thus, as shown by MÖCK, X-ray transmission topograms from Si doped GaAs will display a two-fold pseudo-symmetry only.

The true crystallographic symmetry of the set of majority type dislocation bundles is, however, as experimentally determined for majority type dislocation bundles in undoped GaAs [MÖCK] and predicted by CURIE's symmetry principle, the point symmetry group $42 \mathrm{~m}$ in both cases, undoped and Si doped GaAs. Since this is a consequence of the applicable deformation geometry [REID], we expect the crystallographic parameters of some of the minority type dislocation bundles to possess the same symmetry properties. Minority type dislocation bundles of the first type at the main wafer orientation flat, on the other hand, are expected to possess only the point symmetry group $\mathrm{m}$ since they are caused by an asymmetric disturbance of the applicable deformation geometry. 


\section{Assumption about the values of the actually encountered strains}

The average value of $\varepsilon^{\text {cri }}$ is obtained by the relation $\varepsilon^{\text {cri }}=1 / 2\left\{\sigma^{\text {cri }} /\left(\mathrm{c}_{11}+2 \mathrm{c}_{12}\right)+\sigma^{\text {cri }} / 2 \mathrm{c}_{44}\right\}$. With the "critical stress for slip defect generation" $\left(\sigma^{\text {cri }}\right)$ given by KIYAMA et al for $650{ }^{\circ} \mathrm{C}$, we obtain $\varepsilon^{\text {cri }}=4.7410^{-}$

5. The residual strains $\varepsilon^{\text {res }}$ at room temperature (see Figs. 1a and 3a,b) are due to the presence of the thermal treatment induced dislocation bundles and are at the wafer margin of about the same magnitude as $\varepsilon^{\text {cri }}$. Since the actually encountered strains $\varepsilon^{\text {act }}$ must have been significantly higher that the critical strain $\varepsilon^{\text {cri }}$ we make the assumption $\varepsilon^{\text {act }}=2 \varepsilon^{\text {res }}+\varepsilon^{\text {cri }}$. The usage of the factor 2 in this relation is based on the speculation below.

We consider $\varepsilon^{\text {act }}$ as to be mainly the result of dislocation pairs in the majority type dislocation bundles. Dislocation pairs were formed at high temperatures because they relieved the thermal treatment induced strains more effectively that unpaired dislocations [MÖCK]. At room temperature, on the other hand, the higher strain relief efficiency of dislocation pairs is considered to lead to lower residual strains than one would expect for unpaired dislocations.

\section{Resolved thermal shear stresses on dislocation pairs}

Applying the appropriate deformation geometry [REID], it has been shown for a dislocation pair on the same glide plane that forces with an equal magnitude but opposite sign are acting on the $60^{\circ}$ segments [MÖCK]. These forces compensate each other and the $60^{\circ}$ segments of a dislocation pair are effectively motionless in any direction that is not parallel to their line direction. After a pair has been formed, the movement of a dislocation pair is solely driven by the forces that are acting on the screw segments. In terms of a force balance, the driving force on a dislocation pair is equivalent to the sum of the Peach-Koehler forces on the screw segments of its constituent dislocations [MÖCK].

\section{Justification for the usage of the "critical stress for slip defect generation"}

We use the experimentally obtained "critical stress for slip defect generation", below referred to as critical thermal stress for dislocation generation, in GaAs wafers [KIYAMA et al.] for our model rather than the critical resolved shear stress as used by SAWADA et al. (and as employed extensively for estimations of 
thermally induced plastic deformation in GaAs bulk crystals during the growth process and the subsequent cooling down to room temperature, e.g., JORDAN et al.). For the following reasons, we consider the former concept to be more appropriate for our model.

The former stress threshold was derived by KIYAMA et al. and its temperature dependence determined for exactly the same deformation geometry [REID]. This implies that the same eight slip systems were operating during KIYAMA's and co-workers' experiments simultaneously as we observed for our wafers [MÖCK].

The critical resolved shear stress, however, is usually derived form experiments that were specifically designed for only one slip system to operate or, at least, to dominate [VÖLKL].

Since the onset of plastic deformation was determined by KIYAMA et al. as the first occurrence of slip lines (i.e. visible features of minority type dislocation bundles) on the wafer surface, this particular stress threshold is supposed to include other stress thresholds such as the ones for heterogeneous dislocation nucleation in $\langle 110\rangle$ peripheral areas, possible dislocation interactions, and multiplication for the applicable deformation geometry [REID].

It is well known that plastic deformation is a time dependent process. Consequently, any critical stress threshold is strain rate dependent [ALEXANDER and HAASEN]. Although the strain rate is neither for our experiments nor for the experiments by KIYAMA et al. known, it is reasonable to assume that it was similar in both cases since the same deformation method and geometry [REID] were employed.

In summary, it is, thus, more appropriate to use this value for our model rather than any of the critical resolved shear stress values from the literature, which differ considerably to each other and to the critical thermal stress for dislocation generation.

\section{Naturally occurring distribution of dislocation sources}

On the basis of FITZGERALD's experimental observations on the stability of differently shaped strained (In,Ga)As mesas against plastic deformation by dislocations glide from the side walls during thermal treatments, we believe that surface-step bunching is one of the crucial factors which determines the spatial distribution of nucleation sites for majority type dislocations bundles. Surface step bunching occurs readily at III-V compound semiconductor surfaces around the $\langle 100\rangle$ orientation at temperatures used for epitaxial 
growth and can result in facets of $\mu \mathrm{m}$ dimensions [e.g., HEY et. al.]. Facets, on the other hand, have been shown to be preferential nucleation sides of dislocation bundles if sufficiently high thermal stresses are present [BILLIG].

Dr. Peter Möck

Department of Materials

University of Oxford

Parks Road

Oxford OX1 3PH

England, U.K.

now at:

Department of Physics (M/C 273)

University of Illinois at Chicago

845 West Taylor Street

Chicago, IL 60607-7059

U.S.A.

peter_moeck@hotmail.com 